\title{
Pacific
}

Journal of

Mathematics

\section{SEMI-TOPOLOGICAL CYCLE THEORY I}

JYH-HAUR TEH

Volume 259 No. 1

September 2012 


\title{
SEMI-TOPOLOGICAL CYCLE THEORY I
}

\author{
JYH-HAUR TEH
}

\begin{abstract}
We study algebraic varieties parametrized by topological spaces and enlarge the domain of Lawson homology and morphic cohomology to this category. We prove a Lawson suspension theorem and a splitting theorem. A version of the Friedlander-Lawson moving lemma is obtained to prove a duality theorem between Lawson homology and morphic cohomology for smooth semi-topological projective varieties. $K$-groups for semi-topological projective varieties and Chern classes are also constructed.
\end{abstract}

\section{Introduction}

Algebraic cycles are basic ingredients in studying invariants of algebraic varieties. The collection of all $r$-dimensional algebraic cycles of a projective variety $X$ forms a topological abelian group $Z_{r}(X)$. Lawson [1989] studied these groups from a homotopical viewpoint and proved a suspension theorem that serves as a cornerstone for Lawson homology and morphic cohomology developed later [Friedlander 1991; Friedlander and Lawson 1992; 1997; 1998]. A continuous map $f: S^{n} \rightarrow Z_{r}(X)$ from the $n$-sphere to $Z_{r}(X)$ can be viewed as a family of algebraic cycles parametrized by $S^{n}$. This family can also be considered as an "algebraic cycle" on $X \times S^{n}$. This motivates us to consider algebraic varieties parametrized by topological spaces and consider algebraic cycles on them.

When the base space is an algebraic variety and the parametrization is algebraic, this is just the relative theory of algebraic varieties. The main point of our study is that our base spaces are usually very general topological spaces, so their rings of continuous complex-valued functions are not Noetherian. This makes classical algebraic methods difficult to apply. It is well known in the algebraic case when we wish a family of algebraic varieties to behave well we need the family to be flat over the base scheme. The flatness of a family of varieties is equivalent to the property that the family is the pull back of the universal family over a Hilbert scheme by an algebraic morphism to the Hilbert scheme. So to obtain a nice theory, we define our "semi-topological variety" to be a continuous map from a topological space $S$

MSC2010: primary 14C25; secondary 19E15.

Keywords: Lawson homology, morphic cohomology, semi-topological varieties,

Friedlander-Lawson moving lemma, Hilbert scheme, Chow varieties. 
to some Hilbert scheme with some additional technical assumptions. We are able to define semi-topological algebraic cycles on semi-topological projective varieties and extend the definition of Lawson homology and morphic cohomology to them. This paper is the first part of this theory. A Hodge theory and a Riemann-Roch theorem will be given in a forthcoming paper.

Let us give a brief overview of this paper. In Section 2, we define semi-topological projective varieties and algebraic cycles on them. Some basic topological properties of semi-topological cycle groups are studied. In Section 3, we prove the Lawson suspension theorem and a splitting theorem for semi-topological projective varieties. In Section 4, we give a version of the Friedlander-Lawson moving lemma for semi-topological projective varieties and use it to prove a duality theorem between the Lawson homology and morphic cohomology for semi-topological smooth projective varieties. In Section 5, we compute the Lawson homology group of divisors in a semi-topological smooth projective variety. In Section 6, we construct $K$-groups and Chern classes.

\section{Semi-topological varieties}

Let us briefly recall the construction of cycle groups of complex projective varieties. For a complex projective variety $X$, we write $\mathscr{C}_{r, d}(X)$ for the collection of all effective $r$-cycles of degree $d$ on $X$. According to the Chow theorem, $\mathscr{C}_{r, d}(X)$ is a projective variety. Let $\mathscr{C}_{r}(X)=\bigsqcup_{d \geq 0} \mathscr{C}_{r, d}(X)$ be the Chow monoid and $Z_{r}(X)=\left[\mathscr{C}_{r}(X)\right]^{+}$be the naive group completion of $\mathscr{C}_{r}(X)$. Let

$$
K_{r, d}(X)=\coprod_{d_{1}+d_{2} \leq d} \pi\left(\mathscr{C}_{r, d_{1}}(X) \times \mathscr{C}_{r, d_{2}}(X)\right),
$$

where $\pi: \mathscr{b}_{r}(X) \times \mathscr{b}_{r}(X) \rightarrow Z_{r}(X)$ is the map $(a, b) \mapsto a-b$. We have a filtration

$$
K_{r, 0}(X) \subseteq K_{r, 1}(X) \subseteq \cdots=Z_{r}(X) .
$$

Each $K_{r, d}(X)$ is compact and the topology of $Z_{r}(X)$ is the weak topology induced from this filtration. With this topology, $Z_{r}(X)$ is a topological abelian group. If $Y$ is also a projective variety, we write $Z_{r}(Y)(X)$ for the group of algebraic $r$ cocycles on $X$ with values in $Y$, that is, $c \in Z_{r}(Y)(X)$ if $c \in Z_{r+k}(X \times Y)$ where $k$ is the dimension of $X$, the projection from $c$ to $X$ is surjective and fibers of $c$ over $X$ are $r$-cycles in $Y$.

Throughout this paper, $S$ is a compact topological space with base point $s_{0}$. We write $\mathbb{P}_{S}^{n}$ for $\mathbb{P}^{n} \times S$.

Definition 2.1. A semi-topological projective variety over $S$ is a continuous map $\mathbb{X}: S \rightarrow \operatorname{Hil}_{p} \mathbb{P}^{n}$ such that $\mathbb{X}_{s}:=\mathbb{X}(s)$ is a normal projective variety for all $s \in S$, where $\operatorname{Hil}_{p} \mathbb{P}^{n}$ is the Hilbert scheme of $\mathbb{P}^{n}$ associated to a Hilbert polynomial $p$. 
We write $\mathbb{X} \subset \mathbb{P}_{S}^{n}$ in this case. We define the dimension of $\mathbb{X}$ to be the dimension of $\mathbb{X}_{s_{0}}$, and write $\left|\mathbb{X}_{s_{0}}\right|$ for the algebraic variety corresponding to $\mathbb{X}_{s_{0}}$.

Definition 2.2. Suppose that $\mathbb{X} \subset \mathbb{P}_{S}^{n}, \mathbb{Y} \subset \mathbb{P}_{S}^{m}$ are semi-topological projective varieties over $S$. Let

$$
Z_{r}(\mathbb{Y})(\mathbb{X}):=\left\{\alpha \in \operatorname{Map}\left(\left(S, s_{0}\right),\left(Z_{k}\left(\mathbb{P}^{n} \times \mathbb{P}^{m}\right), 0\right)\right) \mid \alpha(s) \in Z_{r}\left(\mathbb{Y}_{s}\right)\left(\mathbb{X}_{s}\right)\right\},
$$

where $k=r+\operatorname{dim} \mathbb{X}$. Let $Z_{k, \leq e}\left(\mathbb{P}^{n} \times \mathbb{P}^{m}\right)$ be the image of

$$
\coprod_{e_{1}+e_{2} \leq e} \mathscr{C}_{k, e_{1}}\left(\mathbb{P}^{n} \times \mathbb{P}^{m}\right) \times \mathscr{C}_{k, e_{2}}\left(\mathbb{P}^{n} \times \mathbb{P}^{m}\right)
$$

in $Z_{k}\left(\mathbb{P}^{n} \times \mathbb{P}^{m}\right)$. The topology of $Z_{k}\left(\mathbb{P}^{n} \times \mathbb{P}^{m}\right)$ is the weak topology induced from the filtration

$$
Z_{k, \leq 0}\left(\mathbb{P}^{n} \times \mathbb{P}^{m}\right) \subseteq Z_{k, \leq 1}\left(\mathbb{P}^{n} \times \mathbb{P}^{m}\right) \subseteq \cdots=Z_{k}\left(\mathbb{P}^{n} \times \mathbb{P}^{m}\right)
$$

Let $Z_{r, \leq e}(\mathbb{Y})(\mathbb{X}):=\operatorname{Map}\left(\left(S, s_{0}\right),\left(Z_{k, \leq e}\left(\mathbb{P}^{n} \times \mathbb{P}^{m}\right), 0\right)\right) \cap Z_{r}(\mathbb{Y})(\mathbb{X})$. We equip $\operatorname{Map}\left(\left(S, s_{0}\right),\left(Z_{k, \leq e}\left(\mathbb{P}^{n} \times \mathbb{P}^{m}\right), 0\right)\right)$ with the compact-open topology and we give $Z_{r, \leq e}(\mathbb{Y})(\mathbb{X})$ the subspace topology. From the filtration

$$
Z_{r, \leq 0}(\mathbb{Y})(\mathbb{X}) \subseteq Z_{r, \leq 1}(\mathbb{Y})(\mathbb{X}) \subseteq \cdots=Z_{r}(\mathbb{Y})(\mathbb{X})
$$

we equip $Z_{r}(\mathbb{Y})(\mathbb{X})$ with the weak topology.

Then it is not difficult to see that $Z_{r}(\mathbb{Y})(\mathbb{X})$ is a topological abelian group.

Definition 2.3. If $\mathbb{Y}, \mathbb{Y}^{\prime}$ are two semi-topological projective varieties, we say that $\mathbb{Y}^{\prime}$ is a subvariety of $\mathbb{Y}$, denoted by $\mathbb{Y}^{\prime} \subseteq \mathbb{Y}$, if $\mathbb{Y}_{s}^{\prime} \subseteq \mathbb{Y}_{s}$ for all $s \in S$. We write $\mathbb{Y}-\mathbb{Y}^{\prime}$ for the assignment $\left(\mathbb{Y}-\mathbb{Y}^{\prime}\right)(s):=\mathbb{Y}_{s}-\mathbb{Y}_{s}^{\prime}$ for $s \in S$ and call $\mathbb{Y}-\mathbb{Y}^{\prime}$ a semi-topological Zariski open set of $\mathbb{Y}$.

Definition 2.4. Suppose $\mathbb{X} \subset \mathbb{P}_{S}^{n}$, $\mathbb{} \subset \mathbb{P}_{S}^{m}$. Suppose for each $s \in S, f_{s}: \mathbb{X}_{s} \rightarrow \mathbb{Y}_{s}$ is a given morphism of projective varieties. The assignment $s \mapsto f_{s}$ is said to be a morphism between $\mathbb{X}$ and $\mathbb{Y}$, and we write $f: \mathbb{X} \rightarrow \mathbb{Y}$, if $s \mapsto \operatorname{gr} f_{s} \in \mathscr{C}_{r}\left(\mathbb{P}^{n} \times \mathbb{P}^{n}\right)$ is continuous, where gr $f_{s}$ is the graph of $f_{s}$.

Definition 2.5. If $f: \mathbb{X} \rightarrow \mathbb{Y}$ is a morphism of semi-topological projective varieties, define $f_{*}: Z_{r}(\mathbb{X})(\mathbb{W}) \rightarrow Z_{r}(\mathbb{Y})(\mathbb{W})$ by

$$
\left(f_{*} \alpha\right)(s):=q_{s *}\left(\left(\mathbb{W}_{s} \times \operatorname{gr} f_{s}\right) \cdot p_{s}^{*}(\alpha(s))\right),
$$

where $p: \mathbb{W} \times \mathbb{X} \times \mathbb{Y} \rightarrow \mathbb{W} \times \mathbb{X}, q: \mathbb{W} \times \mathbb{X} \times \mathbb{Y} \rightarrow \mathbb{W} \times \mathbb{Y}$ are projections.

Proposition 2.6. The map $f_{*}$ is continuous. 
Proof. First, $p_{s}^{*}$ and $q_{s *}$ are clearly continuous and that $\left(\mathbb{W}_{s} \times \operatorname{gr} f_{s}\right)$ meets $p_{s}^{*}(\alpha(s))$ properly. It follows that the intersection product on cycles intersecting properly is continuous [Fulton 1998]. Thus $f_{*}$ is continuous.

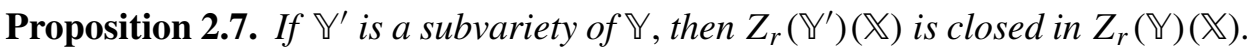

Proof. For $\alpha \in Z_{r, \leq e}(\mathbb{Y})(\mathbb{X})-Z_{r, \leq e}\left(\mathbb{Y}^{\prime}\right)(\mathbb{X})$, there is $s_{1} \in S$ such that

$$
\alpha\left(s_{1}\right) \in Z_{r, \leq e}\left(\mathbb{Y}_{s_{1}}^{\prime}\right)\left(\mathbb{X}_{s_{1}}\right)-Z_{r, \leq e}\left(\mathbb{Y}_{s_{1}}^{\prime}\right)\left(\mathbb{X}_{s_{1}}\right) .
$$

Since $Z_{r, \leq e}\left(\mathbb{Y}_{s_{1}}^{\prime}\right)\left(\mathbb{X}_{s_{1}}\right)$ is closed in $Z_{r, \leq e}\left(\mathbb{Y}_{s_{1}}\right)\left(\mathbb{X}_{s_{1}}\right)$ [Teh 2010, Proposition 2.9], there is a $V$ open in $Z_{k, \leq e}\left(\mathbb{P}^{n} \times \mathbb{P}^{m}\right)$ such that

$$
\alpha\left(s_{1}\right) \in V \cap Z_{r, \leq e}\left(\mathbb{Y}_{s_{1}}\right)\left(\mathbb{X}_{s_{1}}\right) \subset Z_{r, \leq e}\left(\mathbb{Y}_{s_{1}}\right)\left(\mathbb{X}_{s_{1}}\right)-Z_{r, \leq e}\left(\mathbb{Y}_{s_{1}}^{\prime}\right)\left(\mathbb{X}_{s_{1}}\right) .
$$

Let

$$
W=\left\{\beta \in \operatorname{Map}\left(\left(S, s_{0}\right),\left(Z_{k, \leq e}\left(\mathbb{P}^{n} \times \mathbb{P}^{m}\right), 0\right)\right) \mid \beta\left(s_{1}\right) \in V\right\} .
$$

Then $W$ is open in $\operatorname{Map}\left(\left(S, s_{0}\right),\left(Z_{k, \leq}\left(\mathbb{P}^{n} \times \mathbb{P}^{m}\right), 0\right)\right)$ and

$$
\alpha \in W \cap Z_{r, \leq e}(\mathbb{Y})(\mathbb{X}) \subseteq Z_{r, \leq e}(\mathbb{Y})(\mathbb{X})-Z_{r, \leq e}\left(\mathbb{Y}^{\prime}\right)(\mathbb{X}) .
$$

Therefore, the set $Z_{r, \leq e}(\mathbb{Y})(\mathbb{X})-Z_{r, \leq e}\left(\mathbb{Y}^{\prime}\right)(\mathbb{X})$ is open and $Z_{r, \leq e}\left(\mathbb{Y}^{\prime}\right)(\mathbb{X})$ is closed in $Z_{r, \leq e}(\mathbb{Y})(\mathbb{X})$. We then have that $Z_{r}\left(\mathbb{Y}^{\prime}\right)(\mathbb{X})$ is closed in $Z_{r}(\mathbb{Y})(\mathbb{X})$.

Recall that there is a functor $k$ constructed by Steenrod [1967] from the category of topological spaces to the category of compactly generated spaces that acts like a retraction. Furthermore, for any topological space $X, X$ and $k(X)$ have the same homology and homotopy groups. Recall that by the construction in [Teh 2008], if $H$ is a normal closed subgroup of $G$ and both are compactly generated, then the short exact sequence $0 \rightarrow H \rightarrow G \rightarrow G / H \rightarrow 0$ gives a fibration

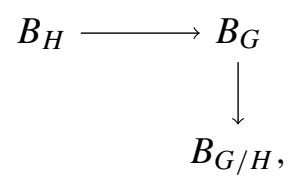

where $B G$ is the classifying space of $G$. Thus we have a long exact sequence of homotopy groups

$$
\cdots \rightarrow \pi_{n}(H) \rightarrow \pi_{n}(G) \rightarrow \pi_{n}(G / H) \rightarrow \pi_{n-1}(H) \rightarrow \cdots .
$$

Combined with the Steenrod functor $k$, once we have some complicated topological abelian groups that form the short exact sequence stated above, we get a long exact sequence of homotopy groups. The following is an application of this result. 
Definition 2.8. Let

$$
Z_{r}\left(\mathbb{Y} ; \mathbb{Y}^{\prime}\right)(\mathbb{X}):=\frac{Z_{r}(\mathbb{Y})(\mathbb{X})}{Z_{r}\left(\mathbb{Y}^{\prime}\right)(\mathbb{X})} \quad \text { and } \quad Z^{t}(\mathbb{X}):=Z_{r}\left(\mathbb{P}_{S}^{t} ; \mathbb{P}_{S}^{t-1}\right)(\mathbb{X})
$$

where $\mathbb{Y}^{\prime}$ is a semi-topological subvariety of $\mathbb{Y}$.

Corollary 2.9. We have a long exact sequence of homotopy groups

$\cdots \rightarrow \pi_{n} Z_{r}\left(\mathbb{Y}^{\prime}\right)(\mathbb{X}) \rightarrow \pi_{n} Z_{r}(\mathbb{Y})(\mathbb{X}) \rightarrow \pi_{n} Z_{r}\left(\mathbb{Y} ; \mathbb{Y}^{\prime}\right)(\mathbb{X}) \rightarrow \pi_{n-1} Z_{r}\left(\mathbb{Y}^{\prime}\right)(\mathbb{X}) \rightarrow \cdots$

Definition 2.10. Let $p t: S \rightarrow \operatorname{Hil}_{p} \mathbb{P}^{n}$ be a constant map whose image is a point in $\mathbb{P}^{n}$. Then $Z_{r}(\mathbb{Y})(p t)$ is isomorphic to $Z_{r}(\mathbb{Y})\left(p t^{\prime}\right)$ for any two such maps $p t, p t^{\prime}$. We write $Z_{r}(\mathbb{Y}):=Z_{r}(\mathbb{Y})(p t)$ without referring to which point we take. The map $p t$ is called a point map.

Definition 2.11. Define

$$
\mathbb{M}_{S, n}(\mathbb{X}):=\pi_{n} Z_{0}(\mathbb{X}) \quad \text { and } \quad \mathbb{T}_{S}^{n}(\mathbb{X}):=\pi_{2 m-n} Z^{m}(\mathbb{X}),
$$

where $m$ is the dimension of $\mathbb{X}$.

Example 2.12. When $S=S^{0}$ is the 0 -dimensional sphere, and $\mathbb{X}=X \times S^{0}$ for some projective variety $X$, then

$$
\mathbb{H}_{S, n}(\mathbb{X})=\pi_{n} Z_{0}(X) \cong H_{n}(X)
$$

by the Dold-Thom theorem. If $X$ is smooth, then

$$
\mathbb{M}_{S}^{n}(\mathbb{X})=\pi_{2 m-n} Z^{m}(X) \stackrel{F L}{\cong} \pi_{2 m-n} Z_{0}(X) \cong H_{2 m-n}(X) \stackrel{P D}{\cong} H^{n}(X),
$$

where $F L$ and $P D$ are the Friedlander-Lawson and Poincaré duality isomorphisms.

\section{Suspension theorem and splitting theorem}

Let us recall that if $X \subseteq \mathbb{P}^{n}$ and $x_{\infty} \in \mathbb{P}^{n+1} \backslash \mathbb{P}^{n}$, the suspension $\sharp X$ of $X$ with respect to $x_{\infty}$ is the join of $X$ and $x_{\infty}$.

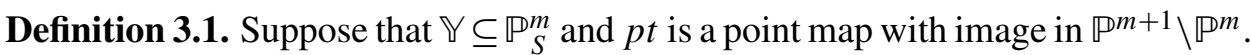
The suspension of $\mathbb{Y}$ with respect to $p t$ is the semi-topological subvariety

$$
\left(\mathbb{Z}_{\mathrm{pt}} \mathbb{Y}\right)(s):=\mathbb{Z}_{\mathrm{pt}(s)} \mathbb{Y}_{s}
$$

So we have $\mathbb{p}_{\mathrm{pt}} \bigvee \subseteq \mathbb{P}_{S}^{m+1}$. The suspension induces a map

$$
Z_{\mathrm{pt}}: Z_{k}\left(\mathbb{P}^{n} \times \mathbb{P}^{m}\right) \longrightarrow Z_{k+1}\left(\mathbb{P}^{n} \times \mathbb{P}^{m+1}\right)
$$

by suspending $\mathbb{P}^{m}$. Hence for each $\alpha \in Z_{r}(\mathbb{Y})(\mathbb{X}), \mathbb{Z}_{\mathrm{pt}}$ induces a semi-topological cycle in $Z_{r+1}(\mathbb{Z})(\mathbb{X})$ by

$$
\left(\mathbb{p}_{\mathrm{pt}} \alpha\right)(s):=\mathbb{Z}_{\mathrm{pt}} \alpha(s)
$$


Theorem 3.2. Let $p t=[0: \ldots: 0: 1] \in \mathbb{P}^{m+1}, \mathbb{Y} \subseteq \mathbb{P}_{S}^{m}$, and $\mathbb{X} \subseteq \mathbb{P}_{S}^{n}$. Then $\mathbb{Z}_{\mathrm{pt*}}: Z_{r}(\mathbb{Y})(\mathbb{X}) \rightarrow Z_{r+1}\left(\mathbb{Z}_{\mathrm{pt}} \mathbb{Y}\right)(\mathbb{X})$ is a weak homotopy equivalence.

Proof. We write $\&$ for $\bigotimes_{\mathrm{pt}}$. Let

$$
T_{r+1}(\mathbb{Z})(\mathbb{X}):=\left\{\begin{array}{l|l}
\alpha \in Z_{r+1}(\mathbb{Y})(\mathbb{X}) & \begin{array}{l}
\alpha(s) \text { meets } \mathbb{X}_{s} \times \mathbb{Y}_{s} \text { properly } \\
\text { in } \mathbb{X}_{s} \times \mathbb{Z} \mathbb{Y}_{s}, \text { for all } s \in S
\end{array}
\end{array}\right\}
$$

Let $\Lambda \subseteq \mathbb{P}^{m+1} \times \mathbb{P}^{1} \times \mathbb{P}^{m+1}$ be the closed subvariety constructed in [Friedlander 1991, Proposition 3.2], which is a geometric description of Lawson's holomorphic taffy. Let $\Lambda_{t}:=\Lambda \bullet\left(\mathbb{P}^{m+1} \times\{t\} \times \mathbb{P}^{m+1}\right), t \in \mathbb{C}$. Then for $\alpha \in T_{r+1}(\mathbb{Z})(\mathbb{X})$,

$$
\Phi_{t}(\alpha):=q_{t *}\left(p_{t}^{*} \alpha \cdot\left(\mathbb{P}^{n} \times \Lambda_{t}\right)\right) \in T_{r+1}(\mathbb{Z})(\mathbb{X}),
$$

where

$$
\begin{aligned}
& p_{t}: \mathbb{P}^{n} \times \mathbb{P}^{m+1} \times\{t\} \times \mathbb{P}^{m+1} \rightarrow \mathbb{P}^{n} \times \mathbb{P}^{m+1}, \\
& q_{t}: \mathbb{P}^{n} \times \mathbb{P}^{m+1} \times\{t\} \times \mathbb{P}^{m+1} \rightarrow \mathbb{P}^{n} \times \mathbb{P}^{m+1},
\end{aligned}
$$

are the projections to the first and second, and to the first and fourth factors, respectively. If $t=0$, then $\Phi_{0}(\alpha) \in \mathbb{Z} Z_{r}(\mathbb{Y})(\mathbb{X})$. It is not difficult to see that $\Phi$ is a strong deformation retract of $T_{r+1}(\mathbb{Z})(\mathbb{X})$ to $\mathbb{Z} Z_{r}(\mathbb{Y})(\mathbb{X})$.

Let $x_{1}=[0: \ldots: 0: 1] \in \mathbb{P}^{m+2}$ and $x_{2}=[0: \ldots: 0: 1: 1] \in \mathbb{P}^{m+2}$. Recall that by [Friedlander and Lawson 1998, Proposition 2.3], for any $d>0$, there is an $e(d)>0$ such that for any $e>e(d)$, there is a line $L_{e}$ in $\mathscr{C}_{m+1, e}\left(\mathbb{P}^{m+2}\right)$ containing $e \mathbb{P}^{m+1}$ such that we have a map

$$
\Psi_{e}: Z_{r+1, \leq d}\left(\mathbb{P}^{m+1}\right) \times L_{e} \rightarrow Z_{r+1, \leq d e}\left(\mathbb{P}^{m+1}\right), \quad(Z, D) \mapsto p_{2 *}\left(\left(x_{1} \# Z\right) \bullet D\right),
$$

where $p_{2}: \mathbb{P}^{m+2}-\left\{x_{2}\right\} \rightarrow \mathbb{P}^{m+1}$ is the projection with center $\left\{x_{2}\right\}$. Furthermore, for $D \in L_{e}-\left\{e \mathbb{P}^{m}\right\}, \Psi_{e}(Z, D) \in T_{r+1, d e}\left(\mathbb{P}^{m+1}\right)$ and $\Psi_{e}\left(Z, e \mathbb{P}^{m}\right)=e Z$. When we restrict to cycles having support in $\mathbb{Y} \subseteq \mathbb{P}^{m+1}$, by checking the definition of $\Psi_{e}$, we get a map

$$
\Psi_{e}: Z_{r+1, \leq d}(\mathbb{Y}) \times L_{e} \rightarrow Z_{r+1, \leq d e}(\mathbb{Y})
$$

with the corresponding properties. For $\alpha \in Z_{r+1, \leq d}(\mathbb{Z})(\mathbb{X})$, define

$$
\Psi_{e}(Z, D)(s):=p_{2 *}\left(\left(x_{1} \# \alpha(s)\right) \bullet\left(D \times \mathbb{X}_{s}\right)\right) .
$$

This map is continuous in $s$ and has the same homotopy property as before. Note that if $f: C \rightarrow Z_{r+1}(\mathbb{Y})(\mathbb{X})$ is a map from a compact topological space $C$, the image $\operatorname{Im} f$ is compact and $\operatorname{Im} f \subseteq Z_{k}\left(\mathbb{P}^{n} \times \mathbb{P}^{m}\right)$. By [Teh 2010, Lemma 2.8], $\operatorname{Im} f \subseteq Z_{k, \leq d}\left(\mathbb{P}^{n} \times \mathbb{P}^{m}\right)$ for some $d>0$. Therefore $\operatorname{Im} f \subseteq Z_{r+1, \leq d}(\mathbb{Y})(\mathbb{X})$.

We show that the map $i_{*}: T_{r+1}(\mathbb{Z} \mathbb{Y})(\mathbb{X}) \rightarrow Z_{r+1}(\mathbb{Z} \mathbb{Y})(\mathbb{X})$ induced from the inclusion is a weak homotopy equivalence. Let $[f] \in \pi_{n}\left(Z_{r+1}(\mathbb{Z})(\mathbb{X})\right)$ be a base 
point preserving continuous map. Since $\operatorname{Im} f$ is compact, $\operatorname{Im} f \subseteq Z_{r+1, \leq d}(\mathbb{Z})(\mathbb{X})$ for some $d$. Then by the result above, there is a map

$$
\Psi_{e}: Z_{r+1, \leq d}(\mathbb{Y})(\mathbb{X}) \times L_{e} \rightarrow Z_{r+1, \leq d e}(\mathbb{Z})(\mathbb{X}),
$$

such that $\Psi_{e}\left(f(s), e \mathbb{P}^{m}\right)=f(s)$ and $\Psi_{e}(f(s), D) \in T_{r+1}(\mathbb{Y})(\mathbb{X})$ for all $D$ in $L_{e}-\left\{e \mathbb{P}^{m}\right\}$. Hence $i_{*}\left[\Psi_{e}(f, D)\right]=[f]$, which implies that $i_{*}$ is surjective. For injectivity, if $[g] \in \pi_{n} T_{r+1}(\mathbb{Z})(\mathbb{X})$ is mapped to 0 by $i_{*}$ in $Z_{r+1}(\mathbb{Z})(\mathbb{X})$, then $i_{*} g$ can be extended to a map $\tilde{g}: D^{n+1} \rightarrow Z_{r+1}(\mathbb{Y})(\mathbb{X})$, where $D^{n+1}$ is the unit closed ball. Again, by choosing some $\Psi_{e}$, we can show that $\tilde{g}$ is homotopic to some

$$
\Psi_{e}(\widetilde{g}, D): D^{n+1} \rightarrow T_{r+1}(\mathbb{Y})(\mathbb{X}) .
$$

Thus $[g]=0$. Combining this with the previous result, the proof is complete.

Theorem 3.3 (splitting theorem). If $\mathbb{X}$ is a semi-topological projective variety, there is a map

$$
\xi_{t}: Z_{0}\left(\mathbb{P}^{t}\right)(\mathbb{X}) \rightarrow Z^{t}(\mathbb{X}) \times Z^{t-1}(\mathbb{X}) \times \cdots \times Z^{0}(\mathbb{X})
$$

that is a weak homotopy equivalence.

Proof. Recall that there is an isomorphism $\mathbb{P}^{n} \cong \mathscr{C}_{0, n}\left(\mathbb{P}^{1}\right)$ for any positive integer $n$. The projection map

$$
\mathbb{P}^{t} \cong \mathscr{C}_{0, t}\left(\mathbb{P}^{1}\right) \rightarrow \mathscr{C}_{0,\left(\begin{array}{l}
t \\
k
\end{array}\right)}\left(\mathbb{P}^{k}\right), \quad x_{1}+\cdots+x_{t} \mapsto \sum_{\substack{I \subset\{1, \ldots, t\} \\
|I|=k}} x_{I},
$$

where $x_{I}=x_{i_{1}}+\cdots+x_{i_{t}}$ for $I=\left\{i_{1}, \ldots, i_{t}\right\}$, induces a map

$$
\xi_{t}: Z_{0}\left(\mathbb{P}_{S}^{t}\right)(\mathbb{X}) \rightarrow Z_{0}\left(\mathbb{P}_{S}^{k}\right)(\mathbb{X}) \rightarrow Z^{k}(\mathbb{X})
$$

for $0 \leq k \leq t$. We have a commutative diagram

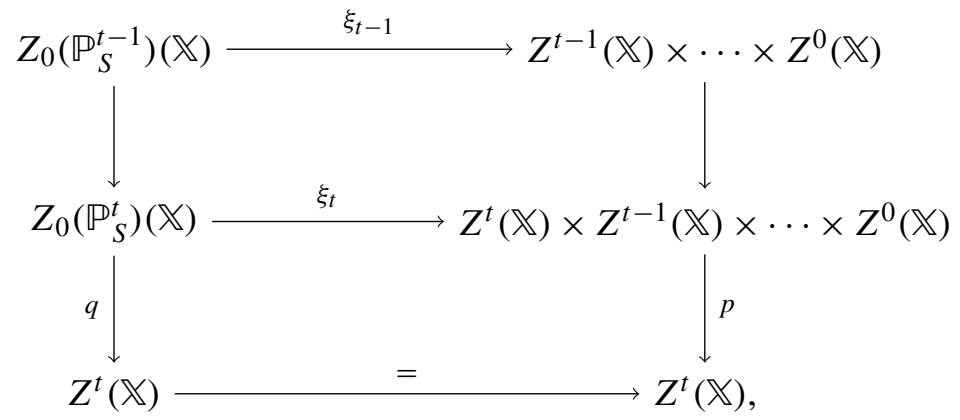

where $q$ is the quotient map and $p$ is the projection map. From the homotopy sequence associated to the vertical columns, we get the result by induction on $t$. 


\section{Moving lemma}

Let $H=\operatorname{Hil}_{p} \mathbb{P}^{n}$ be the Hilbert scheme of $\mathbb{P}^{n}$ associated to the Hilbert polynomial $p$, and let $\pi: \widetilde{H} \longrightarrow H$ be the universal family over $H$. Suppose each algebraic variety parametrized by $H$ is of dimension $m$. Let $U_{\widetilde{H}}(d) \subset \mathbb{P}\left(\Gamma\left(\mathcal{O}_{\mathbb{P}_{H}^{n}}(d)^{m+1}\right)\right)$ be the Zariski open set of those $F=\left(f_{0}, \ldots, f_{m}\right)$ such that

$$
\mathscr{L}_{F}:=\left\{(t, h) \in \mathbb{P}_{H}^{n} \mid F_{h}(t)=0\right\}
$$

misses $\widetilde{H}$, where $F_{h}=\left(f_{0, h}, \ldots, f_{m, h}\right)$ is obtained by pulling back $F$ by the inclusion $z \mapsto(z, h)$ from $\mathbb{P}^{n}$ to $\mathbb{P}_{H}^{n}$. Then $F$ induces a finite morphism $p_{F}: \widetilde{H} \rightarrow \mathbb{P}_{H}^{m}$ by $p_{F}(x):=p_{F_{\pi(x)}}(x)$.

For $Y \in \mathscr{C}_{r, \leq e}(\widetilde{H})(H)$ and $Z \in \mathscr{C}_{\ell, \leq e}(\widetilde{H})(H)$, let

$$
Y \star_{F} Z:=\left\{(y, z) \in Y \times_{H} Z \mid y \neq z, p_{F}(y) \neq p_{F}(z)\right\},
$$

where $Y \times_{H} Z$ is the fiber product of $Y$ and $Z$ over $H$.

By following a similar approach as in [Friedlander and Lawson 1998, Proposition 1.3], we get the following result:

Proposition 4.1. Suppose that $r+\ell \geq m, e \in \mathbb{N}$. There is a Zariski closed subset $\Re_{B}(d)_{e} \subset U_{\widetilde{H}}(d)$ with $\lim _{d \rightarrow \infty}$ Fcodim $\mathscr{B}(d)_{e}=\infty$, where

Fcodim $\mathscr{B}(d)_{e}=\min _{h}\left\{\operatorname{codim} \mathscr{B}(d)_{e, h}\right\} \quad$ and $\mathscr{B}(d)_{e, h}:=\left\{F_{h} \mid F \in \mathscr{B}(d)_{e, h}\right\}$,

such that for any $Y \in \mathscr{C}_{r, \leq e}(\widetilde{H})(H)$ and $Z \in \mathscr{C}_{\ell, \leq e}(\widetilde{H})(H),\left|Y_{h}\right| \star_{F_{h}}\left|Z_{h}\right|$ has pure dimension $r+\ell-m$ whenever $F \in U_{\widetilde{H}}(d)-\mathscr{B}(d)_{e}$.

Now let $\mathbb{X}: S \rightarrow H$ be a semi-topological projective variety. Then the pullback $\mathbb{X}^{*} \mathbb{P}_{H}^{n}=\mathbb{P}_{S}^{n}$ and for $F \in \mathbb{P}\left(\Gamma\left(\mathbb{O}_{\mathbb{P}_{H}^{n}}(d)\right)^{m+1}\right)$, we set $\mathbb{X}^{*} F(x, s):=F(x, \mathbb{X}(s))$ and have $\mathbb{X}^{*} F \in \mathbb{P}\left(\Gamma\left(\mathcal{O}_{\mathbb{P}_{S}^{n}}(d)^{m+1}\right)\right)$. Define $p_{\mathbb{X}^{*} F}: \mathbb{X}^{*}(\widetilde{H}) \rightarrow \mathbb{P}_{H}^{m}$ by

$$
p_{\mathbb{X}^{*} F}(x, s):=p_{F_{\rtimes(s)}}(x),
$$

where $x \in \widetilde{H}, s \in S$. For $\alpha \in Z_{r, \leq e}(\mathbb{X}), \beta \in Z_{\ell, \leq e}(\mathbb{X})$,

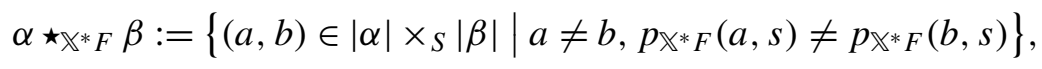

where $|\alpha|$ and $|\beta|$ denote the support of $\alpha$ and $\beta$ respectively.

Let $U_{\widetilde{H}}(d) \subset \mathbb{P}\left(\Gamma\left(\mathscr{O}_{\mathbb{P}_{S}^{n}}(d)^{m+1}\right)\right)$ be the semi-topological Zariski open set of those $F=\left(f_{0}, \ldots, f_{m}\right)$ such that

$$
\mathscr{L}_{F}:=\left\{(t, s) \in \mathbb{P}_{S}^{n} \mid F_{S}(t)=0\right\}
$$

misses $\mathbb{X}^{*} \widetilde{H}$. Then by taking $\widetilde{\mathscr{B}}(d)_{e}=\mathbb{X}^{*}\left(\mathscr{B}(d)_{e}\right)$, from the above result, we also have enough good projections for semi-topological projective varieties when the degree is large enough. 
Corollary 4.2. Let $\mathbb{X} \subseteq \mathbb{P}_{S}^{n}$ be a semi-topological projective variety of dimension $m$. Suppose that $r+\ell \geq m, e \in \mathbb{N}$. There is a semi-topological Zariski closed subset $\widetilde{\mathscr{B}}(d)_{e} \subseteq U_{\mathbb{X}}(d)$ with $\lim _{d \rightarrow \infty}$ Fcodim $\mathscr{B}(d)_{e}=\infty$ such that for $\alpha \in Z_{r, \leq e}(\mathbb{X})$ and $\beta \in Z_{\ell, \leq e}(\mathbb{X}),\left|\alpha_{s}\right| \star_{F_{s}}\left|\beta_{s}\right|$ has pure dimension $r+\ell-m$, where $F \in U_{\mathbb{X}}(d)-\widetilde{\mathscr{B}}(d)_{e}$.

Once we know how to find good projections for semi-topological projective varieties, following an argument in [Friedlander and Lawson 1998], we get a moving lemma for semi-topological projective varieties.

Theorem 4.3. Let $\mathbb{X} \subseteq \mathbb{P}_{S}^{n}$ be a semi-topological projective variety of dimension $m$. Let $r, \ell, e$ be nonnegative integers with $r+\ell \geq m$. Then there exist an open set 0 of $\{0\}$ in $C$ and a continuous map

$$
\widetilde{\Psi}: \mathscr{b}_{\ell}(\mathbb{X}) \times \mathbb{O} \rightarrow \mathscr{C}_{\ell}(\mathbb{X})^{2},
$$

such that $\pi \circ \tilde{\Psi}$ induces by linearity a continuous map

$$
\Psi: Z_{\ell}(\mathbb{X}) \times \mathbb{O} \rightarrow Z_{s}(\mathbb{X})
$$

satisfying the following properties. Let $\psi_{p}=\left.\Psi\right|_{Z_{s}(\mathbb{X}) \times\{p\}}$ for $p \in \mathbb{O}$.

(1) $\psi_{0}=$ Id.

(2) For any $p \in \mathbb{O}, \psi_{p}$ is a continuous group homomorphism.

(3) For any $\alpha \in Z_{\ell, \leq e}(\mathbb{X}), \beta \in Z_{r, \leq e}(\mathbb{X})$, and any $p \neq 0$ in $\mathbb{O}$, each component of excess dimension of the intersection $|\alpha(s)| \cap\left|\psi_{p}(Z)\right|$ is contained in the singular locus of $\left|\mathbb{X}_{s}\right|$, for $s \in S$.

Let $(\mathbb{X}, \mathbb{Y})$ be a pair of semi-topological projective varieties in $\mathbb{P}_{S}^{n}$, where $\mathbb{Y} \subseteq \mathbb{X}$. We say that a map $f:(\mathbb{X}, \mathbb{Y}) \rightarrow\left(\mathbb{X}^{\prime}, \mathbb{Y}^{\prime}\right)$ between two pairs of semi-topological varieties is a relative isomorphism if $f: \mathbb{X} \rightarrow \mathbb{X}^{\prime}$ is a semi-topological morphism such that $f: \mathbb{X}-\mathbb{Y} \rightarrow \mathbb{X}^{\prime}-\mathbb{Y}^{\prime}$ is an isomorphism of semi-topological quasiprojective varieties. The following example is the most important case to us.

Example 4.4. Define $\phi:\left(\mathbb{X} \times{ }_{S} \mathbb{P}_{S}^{t}, \mathbb{X} \times{ }_{S} \mathbb{P}_{S}^{t-1}\right) \rightarrow\left(\mathbb{Z}^{t} \mathbb{X}, \mathbb{P}_{S}^{t-1}\right)$ by

$$
\phi\left(\left(\left[x_{0}: \cdots: x_{n}\right], s\right),\left(\left[a_{0}: \cdots: a_{t}\right], s\right)\right):=\left(\left[a_{0} x_{0}: \cdots: a_{0} x_{n}: a_{1}: \cdots: a_{t}\right], s\right),
$$

where we identify the $\mathbb{P}_{S}^{t-1}$ of the second pair to the hyperplane at infinity of $\rrbracket^{t} \mathbb{X}$. Then it is not difficult to see that $\phi$ is a relative isomorphism.

The following lemma is a special case used in order to define the cycle groups for a quasi-projective variety (see [Lima-Filho 1992]), but it is enough to prove the duality theorem.

Lemma 4.5. Suppose that $f:(\mathbb{X}, \mathbb{Y}) \rightarrow\left(\mathbb{X}^{\prime}, \mathbb{Y}^{\prime}\right)$ is a relative isomorphism, where $\operatorname{dim} \mathbb{Y}^{\prime}<r$, then $Z_{r}(\mathbb{X}) / Z_{r}(\mathbb{Y})$ is weakly homotopic equivalent to $Z_{r}\left(\mathbb{X}^{\prime}\right) / Z_{r}\left(\mathbb{Y}^{\prime}\right)$ and $Z_{r}\left(\mathbb{X}^{\prime}\right) / Z_{r}\left(\mathbb{Y}^{\prime}\right)$ is isomorphic to $Z_{r}\left(\mathbb{X}^{\prime}\right)$. 
Proof. The map $f: \mathbb{X} \rightarrow \mathbb{X}^{\prime}$ induces group homomorphisms $f_{*}: Z_{r}(\mathbb{X}) \rightarrow Z_{r}\left(\mathbb{X}^{\prime}\right)$ and $f_{*}: Z_{r}(\mathbb{Y}) \rightarrow Z_{r}\left(\mathbb{Y}^{\prime}\right)$. Since $f$ restricted to $\mathbb{X}-\mathbb{Y}$ is injective, this gives the injectivity of $f_{*}: Z_{r}(\mathbb{X}) / Z_{r}(\mathbb{Y}) \rightarrow Z_{r}\left(\mathbb{X}^{\prime}\right) / Z_{r}\left(\mathbb{Y}^{\prime}\right)$. But since $r>\operatorname{dim} \mathbb{Y}^{\prime}$, $Z_{r}\left(\mathbb{Y}^{\prime}\right)=\{0\}$. Hence $f_{*}$ is surjective and $Z_{r}\left(\mathbb{X}^{\prime}\right) / Z_{r}\left(\mathbb{Y}^{\prime}\right)=Z_{r}\left(\mathbb{X}^{\prime}\right)$.

By using the moving lemma, we got the following duality theorem that is proved by similar arguments in [Friedlander and Lawson 1997].

Theorem 4.6 (Duality theorem). Suppose that $\mathbb{X} \subseteq \mathbb{P}_{S}^{n}$, $\mathbb{\subseteq} \mathbb{P}_{S}^{k}$, where $\operatorname{dim} \mathbb{X}=m$, and $\mathbb{X}, \mathbb{Y}$ are smooth, that is, each $\mathbb{X}_{s}, \mathbb{Y}_{s}$ is smooth. Then there is a weak homotopic equivalence $i_{*}: \mathbb{Z}_{k}(\mathbb{Y})(\mathbb{X}) \cong \mathbb{Z}_{m+k}(\mathbb{X} \times \mathbb{Y})$, where $i$ is the inclusion.

Corollary 4.7. Suppose that $\mathbb{X}$ is a smooth semi-topological projective variety of dimension $m$. If $0 \leq t \leq m$, then $Z^{t}(\mathbb{X})$ is weakly homotopic equivalent to $Z_{m-t}(\mathbb{X})$. Proof. $Z^{t}(\mathbb{X})=\frac{Z_{0}\left(\mathbb{P}^{t}\right)(\mathbb{X})}{Z_{0}\left(\mathbb{P}^{t-1}\right)(\mathbb{X})} \cong \frac{Z_{m}\left(\mathbb{X} \times \mathbb{P}_{S}^{t}\right)}{Z_{m}\left(\mathbb{X} \times \mathbb{P}_{S}^{t-1}\right)} \cong \frac{Z_{m}\left(\mathbb{Z}^{t} \mathbb{X}\right)}{Z_{m}\left(\mathbb{X} \times \mathbb{P}^{t-1}\right)} \cong Z_{m}\left(\mathbb{Z}^{t} \mathbb{X}\right) \cong Z_{m-t}(\mathbb{X})$.

\section{Semi-topological divisors}

Suppose that the dimension of $\mathbb{X}$ is greater than 0 . Let

$$
K[\mathbb{X}]=\mathscr{C}(S)\left[Z_{0}, \ldots, z_{n}\right] / I(\mathbb{X})=\bigoplus_{d \geq 0} K_{d}(\mathbb{X}),
$$

where $\mathscr{C}(S)$ is the ring of complex-valued continuous functions on $S$, and $K_{d}(\mathbb{X})$ is the collection of homogeneous polynomials of degree $d$ in $K[\mathbb{X}]$.

Proposition 5.1. If $f \in \mathscr{C}(S)\left[z_{0}, \ldots, z_{n}\right]$ is homogenous of degree $d$ and is not the zero polynomial for any $s \in S$, then $f$ defines an effective semi-topological divisor $(f) \in \mathscr{C}_{n-1}\left(\mathbb{P}_{S}^{n}\right)$ by

$$
(f)(s):=\left(f_{s}\right)
$$

for $s \in S$, where $\left(f_{s}\right)$ is the divisor on $\mathbb{P}^{n}$ defined by $f_{s}$.

Proof. Since $f_{s}$ is not the zero polynomial for any $s \in S,\left(f_{s}\right)$ is an effective divisor for any $s \in S$. From the definition of the Chow form, we see that the coefficients of the Chow form $F_{\left(f_{s}\right)}$ of $\left(f_{s}\right)$ are continuous functions of the coefficients of $f$. This implies that the assignment $(f): S \rightarrow \mathscr{C}_{n-1, d}\left(\mathbb{P}^{n}\right)$ is continuous.

Definition 5.2. Let $\mathscr{C}(S)\left[z_{0}, \ldots, z_{n}\right]_{d, \mathbb{X}}$ be the collection of all $f \in \mathscr{C}(S)\left[z_{0}, \ldots, z_{n}\right]$ of degree $d$ such that $\left(f_{s}\right)$ meets $\mathbb{X}_{s}$ properly in $\mathbb{P}^{n}$ for all $s \in S$. For $f+I(\mathbb{X})$ in $K_{d}(\mathbb{X})$, where $f \in \mathscr{C}(S)\left[z_{0}, \ldots, z_{n}\right]_{d, \mathbb{X}}$, let

$$
(f+I(\mathbb{X}))(s):=\left(f_{s}\right) \cdot \mathbb{X}_{s}
$$

for $s \in S$. Then $(f+I(\mathbb{X}))$ is a semi-topological divisor on $\mathbb{X}$. Let

$$
W_{d}(\mathbb{X}):=\left\{(f+I(\mathbb{X})) \mid f \in \mathscr{C}(S)\left[z_{0}, \ldots, z_{n}\right]_{d, \mathbb{X}}\right\}
$$


Let $W(\mathbb{X})=\bigsqcup_{d \geq 0} W_{d}(\mathbb{X})$ and

$$
Z_{m-1}(\mathbb{X})^{l i n}=\left\{\alpha-\beta \mid \text { where } \alpha, \beta \in W(\mathbb{X}), \alpha\left(s_{0}\right)=\beta\left(s_{0}\right)\right\}
$$

We say that a semi-topological divisor $D \in Z_{m-1}(\mathbb{X})$ is semi-topologically linearly equivalent to zero if $D \in Z_{m-1}(\mathbb{X})^{l i n}$.

Proposition 5.3. Let $T_{d}(\mathbb{X})=\left\{(f) \mid f \in \mathscr{C}(S)\left[z_{0}, \ldots, z_{n}\right]_{d, \mathbb{X}}\right\}, T(\mathbb{X})=\bigsqcup_{d \geq 0} T_{d}(\mathbb{X})$ and $\widetilde{T}(\mathbb{X}):=\left\{(f)-(g) \mid(f)\left(s_{0}\right)=(g)\left(s_{0}\right),(f),(g) \in T(\mathbb{X})\right\} \subseteq Z_{m-1}(\mathbb{X})$.

(1) $\widetilde{T}(\mathbb{X})$ is isomorphic as a topological group to $Z_{m-1}(\mathbb{X})^{\text {lin }}$.

(2) $\widetilde{T}(\mathbb{X})$ is weakly homotopy equivalent to $Z_{n-1}\left(\mathbb{P}_{S}^{n}\right)$ where $\mathbb{X} \subseteq \mathbb{P}_{S}^{n}$.

Proof. The isomorphism between $\widetilde{T}(\mathbb{X})$ and $Z_{m-1}(\mathbb{X})^{\text {lin }}$ is given by the natural $\operatorname{map}(f)-(g) \mapsto(f+I(\mathbb{X}))-(g+I(\mathbb{X}))$. By the moving lemma, for any $e>0$, there is an integer $e(d)$ such that for any $k>e(d)$ there is a continuous function $\Theta_{k}: Z_{n-1, \leq e}\left(\mathbb{P}^{n}\right) \times \ell^{0} \rightarrow Z_{n-1, k e}\left(\mathbb{P}^{n}\right)$ such that:

(1) $\Theta_{k}(c, 0)=k c$.

(2) $\Theta_{k}(c, t)$ meets $\mathbb{X}_{s}$ properly for $t \in \ell^{0} \backslash\{0\}$.

Then, by following exactly the argument for proving the suspension theorem, we show that the inclusion $i_{*}: \widetilde{T}(\mathbb{X}) \rightarrow Z_{n-1}\left(\mathbb{P}_{S}^{n}\right)$ is a weak homotopy equivalence.

Proposition 5.4. Suppose that $\mathbb{X} \subseteq \mathbb{P}_{S}^{n}$ is a semi-topological variety of dimension $m$. Then $Z_{m-1}(\mathbb{X})^{\text {lin }}$ is weakly homotopy equivalent to $\operatorname{Map}\left(\left(S, s_{0}\right),\left(Z_{0}\left(\mathbb{P}^{1}\right), 0\right)\right)$. In particular,

$$
\pi_{\ell} Z_{m-1}(\mathbb{X})^{l i n}= \begin{cases}H^{2}(S), & \text { if } \ell=0, \\ H^{1}(S), & \text { if } \ell=1, \\ H^{0}(S), & \text { if } \ell=2, \\ 0, & \text { otherwise }\end{cases}
$$

Proof. We have a homeomorphism

$$
\mathscr{C}(S)\left[z_{0}, \ldots, z_{n}\right]_{d} \rightarrow \operatorname{Map}\left(S, \mathbb{C}^{\left(\begin{array}{c}
n+d \\
d
\end{array}\right)}\right), \quad f \mapsto \text { coefficients of } f .
$$

This homeomorphism reduces to a homeomorphism

$$
\mathscr{C}_{n-1, d}\left(\mathbb{P}^{n}\right) \cong \operatorname{Map}\left(S, \mathbb{P}^{\left(\begin{array}{c}
n+d \\
d
\end{array}\right)-1}\right) \cong \operatorname{Map}\left(S, \mathscr{C}_{0,\left(\begin{array}{c}
n+d \\
d
\end{array}\right)-1}\left(\mathbb{P}^{1}\right)\right) .
$$

Thus $Z_{n-1}\left(\mathbb{P}_{S}^{n}\right) \cong \operatorname{Map}\left(\left(S, s_{0}\right),\left(Z_{0}\left(\mathbb{P}^{1}\right), 0\right)\right)$. From the result above, we have weak homotopy equivalences

$$
Z_{m-1}(\mathbb{X})^{l i n} \cong \widetilde{T}(\mathbb{X}) \cong Z_{n-1}\left(\mathbb{P}_{S}^{n}\right) \cong \operatorname{Map}\left(\left(S, s_{0}\right),\left(Z_{0}\left(\mathbb{P}^{1}\right), 0\right)\right)
$$




\section{Chern classes}

Definition 6.1. Suppose that the dimension of a semi-topological variety $\mathbb{X}$ is $k$. Let

$$
\mathscr{C}^{s, 1}\left(\mathbb{P}_{S}^{n}\right)(\mathbb{X}):=\left\{\alpha \in \operatorname{Map}\left(S, \mathscr{C}_{k+n-s}\left(\mathbb{P}^{m} \times \mathbb{P}^{n}\right)\right) \mid \alpha(s) \in \mathscr{C}^{s, 1}\left(\mathbb{P}^{n}\right)\left(\left|\mathbb{X}_{s}\right|\right)\right\},
$$

where $\mathscr{C}^{s, 1}\left(\mathbb{P}^{n}\right)\left(\left|\mathbb{X}_{s}\right|\right):=\mathscr{C}_{n-s, 1}\left(\left|\mathbb{X}_{s}\right| \times \mathbb{P}^{n}\right)$.

By suspension, we have a sequence

$$
\cdots \rightarrow \mathscr{C}^{s, 1}\left(\mathbb{P}^{n}\right)(\mathbb{X}) \rightarrow \mathscr{C}^{s, 1}\left(\mathbb{P}^{n+1}\right)(\mathbb{X}) \rightarrow \mathscr{C}^{s, 1}\left(\mathbb{P}^{n+2}\right)(\mathbb{X}) \rightarrow \cdots
$$

Let

$$
\mathscr{C}^{s, 1}\left(\mathbb{P}^{\infty}\right)(\mathbb{X}):=\lim _{n \rightarrow \infty} \mathscr{C}^{s, 1}\left(\mathbb{P}^{n}\right)(\mathbb{X})
$$

and let

$$
\operatorname{Vect}^{s}(\mathbb{X}):=\left[\mathscr{C}^{s, 1}\left(\mathbb{P}^{\infty}\right)(\mathbb{X})\right]^{+}
$$

be the group completion. Note that we do not fix a base point of $\mathbb{V e c t}^{s}(\mathbb{X})$. Let

$$
\begin{aligned}
\widetilde{\operatorname{Vect}}^{s}(\mathbb{X}) & :=\left\{f-g \in{\mathbb{V _ { e c t }}}^{s}(\mathbb{X}) \mid f_{s_{0}}=g_{s_{0}}\right\}, \\
\widetilde{\operatorname{Vect}^{s}}(\mathbb{X})_{n}: & =\left\{f-g \in \widetilde{\operatorname{Vect}}^{s}(\mathbb{X}) \mid f, g \in \mathscr{C}^{s, 1}\left(\mathbb{P}^{n}\right)(\mathbb{X})\right\} .
\end{aligned}
$$

Then we have sequences and maps

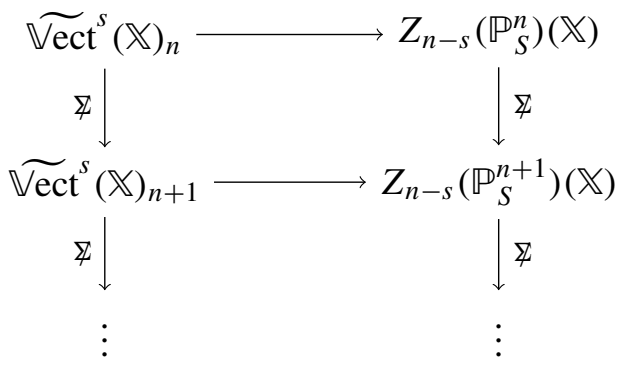

and we get a map

$$
\widetilde{\mathbb{V e c t}^{s}}(\mathbb{X}) \rightarrow \lim _{n \rightarrow \infty} Z_{n-s}\left(\mathbb{P}_{S}^{n}\right)(\mathbb{X})
$$

By taking $\pi_{0}$ on both sides, we get a homomorphism

$$
\pi_{0} \widetilde{\operatorname{Vect}}^{s}(\mathbb{X}) \stackrel{\mathbf{c}}{\longrightarrow} \pi_{0}\left(\lim _{n \rightarrow \infty} Z_{n-s}\left(\mathbb{P}_{S}^{n}\right)(\mathbb{X})\right) \cong \pi_{0} Z_{o}\left(\mathbb{P}_{S}^{n}\right)(\mathbb{X}) \cong \bigoplus_{i=0}^{s} L^{i} H^{2 i}(\mathbb{X})
$$

Definition 6.2. For $[\alpha] \in \pi_{0} \widetilde{\mathbb{V e c t}^{s}}(\mathbb{X}), \mathbf{c}([\alpha]) \in \bigoplus_{i=0}^{s} L^{i} H^{2 i}(\mathbb{X})$ is called the total Chern class of $[\alpha]$. 
The inclusions

$$
\mathscr{C}^{s, 1}\left(\mathbb{P}_{S}^{n}\right)(\mathbb{X}) \hookrightarrow \mathscr{C}^{s+1,1}\left(\mathbb{P}_{S}^{n+1}\right)(\mathbb{X}) \hookrightarrow \mathscr{C}^{s+2,1}\left(\mathbb{P}_{S}^{n+2}\right)(\mathbb{X}) \hookrightarrow \cdots
$$

induce inclusions on

$$
\mathscr{C}^{s, 1}\left(\mathbb{P}_{S}^{\infty}\right)(\mathbb{X}) \hookrightarrow \mathscr{C}^{s+1,1}\left(\mathbb{P}_{S}^{\infty}\right)(\mathbb{X}) \hookrightarrow \mathscr{C}^{s+2,1}\left(\mathbb{P}_{S}^{\infty}\right)(\mathbb{X}) \hookrightarrow \cdots,
$$

which induce again maps on

$$
\widetilde{\mathbb{V e c t}^{s}}(\mathbb{X}) \rightarrow{\widetilde{\mathbb{V e c t}^{s+1}}}^{s}(\mathbb{X}) \rightarrow \widetilde{\mathbb{V e c t}}^{s+2}(\mathbb{X}) \rightarrow \cdots
$$

Let

$$
\widetilde{\operatorname{Vect}}(\mathbb{X}):=\lim _{s \rightarrow \infty} \widetilde{\operatorname{Vect}}^{s}(\mathbb{X}) .
$$

Definition 6.3. Suppose that $\mathbb{X}$ is a semi-topological projective variety. Let

$$
K_{n}(\mathbb{X}):=\pi_{n} \widetilde{\operatorname{Vect}}(\mathbb{X}) .
$$

This is called the $n$-th $K$-group of $\mathbb{X}$.

This construction of Chern classes is a preparation for a proof of a GrothendieckRiemann-Roch theorem for semi-topological projective varieties.

Example 6.4. When $S=S^{0}, \mathbb{X}=X \times S^{0}$ where $X$ is a smooth projective variety. Then

$$
K_{n}(\mathbb{X})=K_{n}^{\text {semi }}(X),
$$

where $K^{n}(X)$ is the semi-topological $K$-group of $X$ constructed by Friedlander and Walker [2002; 2003].

\section{Acknowledgement}

The author thanks Taiwan National Center for Theoretical Sciences (Hsinchu) for proving a nice working environment, and thanks Yu-Wen Kao for her support.

\section{References}

[Friedlander 1991] E. M. Friedlander, "Algebraic cycles, Chow varieties, and Lawson homology", Compositio Math. 77:1 (1991), 55-93. MR 92a:14005 Zbl 0754.14011

[Friedlander and Lawson 1992] E. M. Friedlander and H. B. Lawson, Jr., "A theory of algebraic cocycles", Ann. of Math. (2) 136:2 (1992), 361-428. MR 93g:14013 Zbl 0788.14014

[Friedlander and Lawson 1997] E. M. Friedlander and H. B. Lawson, "Duality relating spaces of algebraic cocycles and cycles”, Topology 36:2 (1997), 533-565. MR 97k:14007 Zbl 0889.14004

[Friedlander and Lawson 1998] E. M. Friedlander and H. B. Lawson, "Moving algebraic cycles of bounded degree”, Invent. Math. 132:1 (1998), 91-119. MR 99k:14011 Zbl 0936.14005

[Friedlander and Walker 2002] E. M. Friedlander and M. E. Walker, "Semi-topological $K$-theory using function complexes”, Topology 41:3 (2002), 591-644. MR 2003g:19005 Zbl 1003.19003 
[Friedlander and Walker 2003] E. M. Friedlander and M. E. Walker, "Rational isomorphisms between $K$-theories and cohomology theories", Invent. Math. 154:1 (2003), 1-61. MR 2004j:19002 Zbl 1025.14002

[Fulton 1998] W. Fulton, Intersection theory, 2nd ed., Ergeb. Math. Grenzgeb. (3) 2, Springer, Berlin, 1998. MR 99d:14003 Zbl 0885.14002

[Lawson 1989] H. B. Lawson, Jr., "Algebraic cycles and homotopy theory", Ann. of Math. (2) 129:2 (1989), 253-291. MR 90h:14008 Zbl 0688.14006

[Lima-Filho 1992] P. Lima-Filho, "Lawson homology for quasiprojective varieties", Compositio Math. 84:1 (1992), 1-23. MR 93j:14007 Zbl 0773.14010

[Steenrod 1967] N. E. Steenrod, "A convenient category of topological spaces", Michigan Math. J. 14 (1967), 133-152. MR 35 \#970 Zbl 0145.43002

[Teh 2008] J.-H. Teh, "Harnack-Thom theorem for higher cycle groups and Picard varieties", Trans. Amer. Math. Soc. 360:6 (2008), 3263-3285. MR 2009a:14011 Zbl 1143.14012

[Teh 2010] J.-H. Teh, "A homology and cohomology theory for real projective varieties", Indiana Univ. Math. J. 59:1 (2010), 327-384. MR 2011g:14018 Zbl 1206.14021

Received January 20, 2010. Revised January 3, 2012.

JYH-HAUR TEH

DEPARTMENT OF MATHEMATICS

NATIONAL TSING HUA UNIVERSITY OF TAIWAN

101 KUANG Fu RoAd HSINCHU 30043

TAIWAN

jyhhaur@math.nthu.edu.tw 


\title{
PACIFIC JOURNAL OF MATHEMATICS
}

\author{
http://pacificmath.org \\ Founded in 1951 by \\ E. F. Beckenbach (1906-1982) and F. Wolf (1904-1989)
}

\section{EDITORS}

V. S. Varadarajan (Managing Editor)

Department of Mathematics

University of California

Los Angeles, CA 90095-1555

pacific@math.ucla.edu

Vyjayanthi Chari

Department of Mathematics

University of California

Riverside, CA 92521-0135

chari@math.ucr.edu

\section{Robert Finn}

Department of Mathematics Stanford University

Stanford, CA 94305-2125

finn@math.stanford.edu

Kefeng Liu

Department of Mathematics

University of California

Los Angeles, CA 90095-1555

liu@math.ucla.edu
Darren Long

Department of Mathematics

University of California

Santa Barbara, CA 93106-3080

long@math.ucsb.edu

Jiang-Hua Lu

Department of Mathematics

The University of Hong Kong

Pokfulam Rd., Hong Kong jhlu@maths.hku.hk

Alexander Merkurjev

Department of Mathematics

University of California

Los Angeles, CA 90095-1555

merkurev@math.ucla.edu
Sorin Popa

Department of Mathematics University of California

Los Angeles, CA 90095-1555 popa@math.ucla.edu

Jie Qing

Department of Mathematics

University of California

Santa Cruz, CA 95064

qing@cats.ucsc.edu

Jonathan Rogawski

Department of Mathematics

University of California

Los Angeles, CA 90095-1555

jonr@math.ucla.edu

\section{PRODUCTION}

pacific@math.berkeley.edu

\section{SUPPORTING INSTITUTIONS}

ACADEMIA SINICA, TAIPEI

CALIFORNIA INST. OF TECHNOLOGY INST. DE MATEMÁTICA PURA E APLICADA KEIO UNIVERSITY

MATH. SCIENCES RESEARCH INSTITUTE NEW MEXICO STATE UNIV.

OREGON STATE UNIV.

\author{
STANFORD UNIVERSITY \\ UNIV. OF BRITISH COLUMBIA \\ UNIV. OF CALIFORNIA, BERKELEY \\ UNIV. OF CALIFORNIA, DAVIS \\ UNIV. OF CALIFORNIA, LOS ANGELES \\ UNIV. OF CALIFORNIA, RIVERSIDE \\ UNIV. OF CALIFORNIA, SAN DIEGO \\ UNIV. OF CALIF., SANTA BARBARA
}

\author{
UNIV. OF CALIF., SANTA CRUZ \\ UNIV. OF MONTANA \\ UNIV. OF OREGON \\ UNIV. OF SOUTHERN CALIFORNIA \\ UNIV. OF UTAH \\ UNIV. OF WASHINGTON \\ WASHINGTON STATE UNIVERSITY
}

These supporting institutions contribute to the cost of publication of this Journal, but they are not owners or publishers and have no responsibility for its contents or policies.

See inside back cover or pacificmath.org for submission instructions.

The subscription price for 2012 is US \$420/year for the electronic version, and \$485/year for print and electronic.

Subscriptions, requests for back issues from the last three years and changes of subscribers address should be sent to Pacific Journal of Mathematics, P.O. Box 4163, Berkeley, CA 94704-0163, U.S.A. Prior back issues are obtainable from Periodicals Service Company, 11 Main Street, Germantown, NY 12526-5635. The Pacific Journal of Mathematics is indexed by Mathematical Reviews, Zentralblatt MATH, PASCAL CNRS Index, Referativnyi Zhurnal, Current Mathematical Publications and the Science Citation Index.

The Pacific Journal of Mathematics (ISSN 0030-8730) at the University of California, c/o Department of Mathematics, 969 Evans Hall, Berkeley, CA 94720-3840, is published monthly except July and August. Periodical rate postage paid at Berkeley, CA 94704, and additional mailing offices. POSTMASTER: send address changes to Pacific Journal of Mathematics, P.O. Box 4163, Berkeley, CA 94704-0163.

PJM peer review and production are managed by EditFLOW ${ }^{\mathrm{TM}}$ from Mathematical Sciences Publishers.

PUBLISHED BY PACIFIC JOURNAL OF MATHEMATICS

at the University of California, Berkeley 94720-3840

A NON-PROFIT CORPORATION

Typeset in LATEX

Copyright $(02012$ by Pacific Journal of Mathematics 


\title{
PACIFIC JOURNAL OF MATHEMATICS
}

\author{
Volume $259 \quad$ No. $1 \quad$ September 2012
}

Extension Theorems for external cusps with minimal regularity

GABRIEL ACOSTA and IGNACIO OJEA

Convergence of axially symmetric volume-preserving mean curvature flow

MARIA ATHANASSENAS and SEVVANDi KANDANAARACHCHI

On the horoboundary and the geometry of rays of negatively curved manifolds

FranÇOISE DAL'bo, Marc PEIGNÉ and ANDREA

SAMBUSETTI

Two infinite versions of the nonlinear Dvoretzky theorem

KEI FUNANO

Nonlocal uniform algebras on three-manifolds

ALEXANDER J. IZZO

Mahlo cardinals and the torsion product of primary abelian groups

PATRICK W. KEEF

Geometry of trinomials

AARON MELMAN

Drinfeld orbifold algebras

ANNE V. SHEPLER and SARAH Witherspoon

Semi-topological cycle theory I

JYH-HAUR TEH

New construction of fundamental domains for certain Mostow groups 209

TiEHONG ZHAO 